\title{
High-resolution phase mapping with parallel Fourier domain optical coherence microscopy for dispersion contrast imaging
}

\author{
Branislav Grajciar, ${ }^{* 1}$ Melanie Herdin, ${ }^{1}$ Cedric Blatter, ${ }^{1}$ Martin Gröschl, ${ }^{2}$ and Rainer A. Leitgeb ${ }^{1}$ \\ ${ }^{1}$ Center for Medical Physics and Biomedical Engineering, Medical University of Vienna, Waehringer Guertel 18-20, \\ A-1090 Vienna, Austria \\ ${ }^{2}$ Institut of Applied Physics, Technical University Vienna, Wiedner Hauptstraße 8-10/E134, A-1040 Vienna, Austria
}

Received November 15, 2011; accepted December 20, 2011; published December 31, 2011

\begin{abstract}
We demonstrate a high-speed parallel line field optical coherence microscopy (OCM) system that exhibits intrinsically high phase stability even with lateral scanning. The presented Fourier domain (FD) OCM system employs a high speed CMOS detector and achieves up to 512000 equivalent A-scans per second. The microscopy system is shown to resolve the smallest elements of the USAF resolution test target (Group 7, Element 6), indicating the transverse (Y- non-scanning parallel direction)) resolution and resolution in $\mathrm{X}$ (scanning) direction $<2.2 \mu \mathrm{m}$. The capability of imaging biological samples is demonstrated on human red blood cells (RBC). The high phase sensitivity allows resolving the thickness map of the RBC. Resolving the phase gives further access to the dispersion properties of the sample, which in turn reveals chemical information of the microsystem.
\end{abstract}

Non-invasive and label-free high spatial/depth resolution real-time (in-vivo) imaging modalities are an emerging need for numerous biomedical applications. Optical coherence tomography (OCT), as one of the well established techniques over the past two decades, enables cross-sectional imaging of tissue microstructure [1]. The transverse resolution of a conventional OCT system can be improved down to sub-micrometer resolution by using high numerical aperture (NA) microscope objectives and thereby it turns OCT to optical coherence microscopy (OCM) [2]. In order to increase the speed and thereby minimize motion artifacts, detection can be parallelized either by full field (FF) or line field (LF) OCT [3-5]. However, increased incoherent scattered light and pixel crosstalk are the main drawbacks of parallel FF detection techniques, which results in lower sensitivity in comparison to truly-confocal (point illumination) OCM. LF OCT, on the other hand, offers reduced pixel crosstalk in one dimension and keeps the speed advantage of a parallel system. In addition, it is shown to exhibit extraordinary phase stability over the parallel direction that can be compared to self-referenced systems [6].

In this paper we introduce line field (LF) FD-OCM that combines the advantage of OCM as a promising technology for high-resolution cellular-level imaging in human tissues with high speed and high phase sensitivity

*E-mail: branislav.grajciar@meduniwien.ac.at (stability) of a parallel FD-OCT system [6]. The phase stability of the present system allows resolving path length differences with sub-nanometer sensitivity similar to spectral domain phase microscopy [7]. Such high sensitivity over a full scanning field offers exciting new perspectives for label-free spectroscopic imaging. Access to the sample dispersion due to the in general complex refractive tissue index allows differentiating biochemical properties and constituents. We demonstrate those advantages on human red blood cells.

\section{Parallel FD-OCM system}

The optical scheme of the parallel FD-OCM system is shown in Fig. 1.

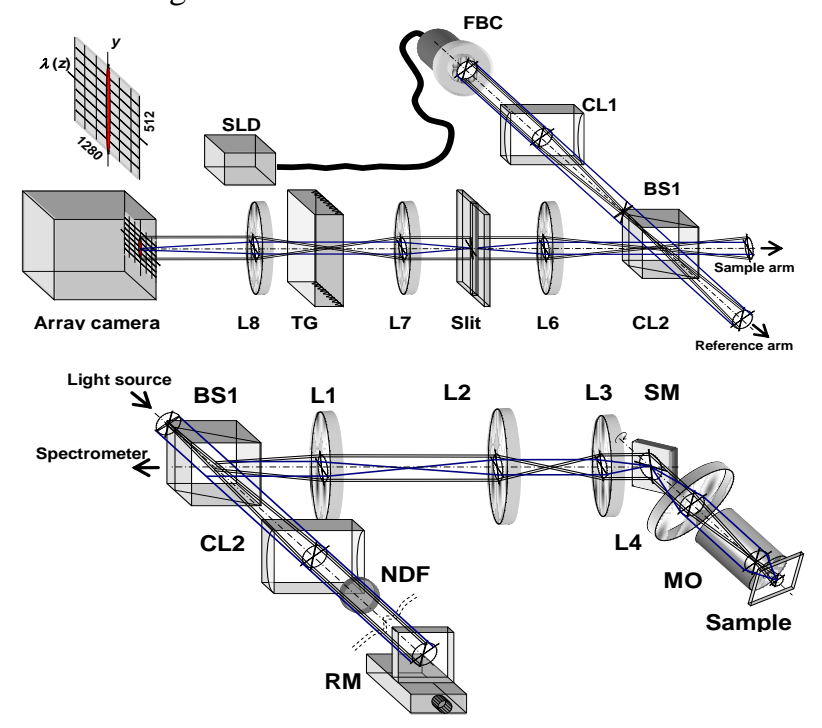

Fig. 1. Schematic of a parallel FD-OCM system. SLD superluminiscent diode; FBC - collimator; CL1, CL2 - cylindrical lenses; L1-7 - achromats; MO- microscope objective; BS1 - nonpolarizing beam splitter ; RM - laser line reference mirror; NDF1 - filter; TG - transmission grating; L8 - imaging lens.

A Michelson interferometer is illuminated by a superluminescent diode (SLD) with a central wavelength of $\lambda_{0}=832 \mathrm{~nm}$ and a spectral full width at half maximum (FWHM) of $\Delta \lambda_{\mathrm{FWHM}}=17 \mathrm{~nm}$. Outgoing light from the SLD 
pigtail is collimated with collimator FBC. The cylindrical lens CL1 defines an anamorphotic scheme and produces line illumination of the sample. The anamorphic beam is split by a non-polarizing beam splitter BS1 into a sample and reference beam. The modification of the sample arm is stated as a main difference from our previously reported parallel FD-OCT system [6]. The lens configuration in the sample arm (L2- achromat with $\mathrm{f}=100 \mathrm{~mm}$ ), L3, L4 achromats with $\mathrm{f}=40 \mathrm{~mm}$ ), MO- microscope objective (Spindler\&Hoyer, 20x, $0.5 \mathrm{NA}$, working distance $1.6 \mathrm{~mm}$ ) offers telecentricity and good imaging performance for extended fields. The theoretical lateral resolution is $1.7 \mu \mathrm{m}$ with a depth of focus of $5.7 \mu \mathrm{m}$. In order to record 3D data sets, a single-axis galvo-scanner is placed in a plane conjugate to the back-focal plane of the MO.

Light beams returning from the sample and reference arms are recombined at the exit of the interferometer. Light is spatially filtered via a slit $(50 \mu \mathrm{m} \times 3 \mathrm{~mm})$ and then fed into the spectrometer. Due to the high spatial coherence of the light source (SLD) used in the system there is a need to suppress spatial coherent crosstalk, which is otherwise degrading the transversal resolution. A slit aperture is positioned at the conjugate plane of the sample to reject light from adjacent voxels, and therefore greatly improving image contrast due to reduced multiply scattered light from outside the coherence volume. However, this ,quasi-confocal' system does not achieve the confocality of a truly-confocal systems, since some light from neighbouring voxels along the illuminated line can mix at the detector. Still, line-scanning OCM (LSOCM), has important advantages over full field OCM (FF-OCM). Line-field illumination reduces pixel crosstalk due to the confocality in the scanning direction, which increases the sensitivity up to more than an order of magnitude. Furthermore, phase-sensitive information is acquired more rapidly, before significant fringe blurring occurs in the case of FD-OCM.

The spectrometer is equipped with a high speed CMOS camera (1280 x $1024 @ 500$ frames/s, 12 $\mu \mathrm{m}$ square pixel, AES Technologie AG). The light is dispersed via transmission grating TG (Wasatch Inc., 1200 lines/mm) and imaged through an achromatic lens $(\mathrm{f}=150 \mathrm{~mm})$ onto horizontal detector lines. The spectral resolution of $0.07 \mathrm{~nm}$ results in a system depth range of $2.7 \mathrm{~mm}$. Due to the low quantum efficiency of the used CMOS detector ( $7 \%$ at $800 \mathrm{~nm})$ and the limited bit depth of 8 bit, a sensitivity of $\sim 74 \mathrm{~dB}$ has been measured at the maximum intensity position with an exposure time of $1 \mathrm{~ms}$.

\section{Measurements of the system resolution}

The en-face resolution performances of the system were verified on an USAF resolution test target (RTT). In order to extract the phase map from an en-face image of the structure, a full 3D data set needs to be recorded. The lateral scanning ( $\mathrm{X}$ axis) was performed using a galvo- scanner. 3D data sets were recorded with at 1000fps (frames per second). Each frame yields after wavelength to wavenumber conversion and FFTs along the spectral coordinate one tomogram consisting of 512 A-scans. Figure 2 shows an en-face intensity image taken at the maximum intensity of the front reflex from the RTT.

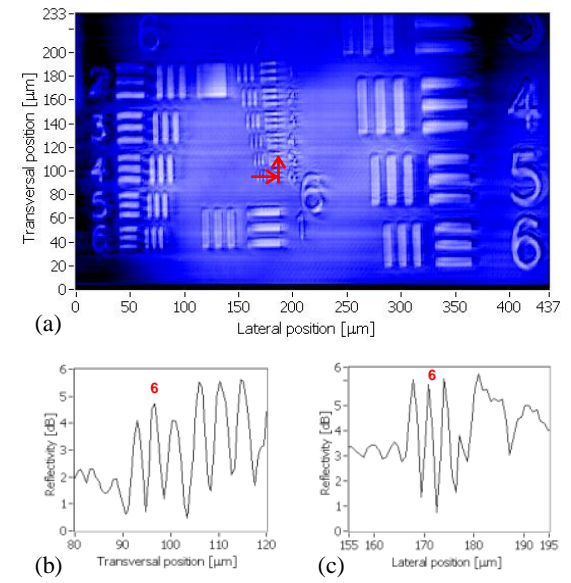

Fig. 2. (a) An en-face intensity map of full field RTT with associated (b) transversal (y) and (c) lateral (x) cross-section along the group 7 , element 6 [indicated by red arrows in (a)], taken at the maximum intensity of the front reflex from the RTT target.

We are able to resolve the smallest element of the USAF resolution test target (Group 7, Element 6), indicating the transverse ( $\mathrm{Y}$ - non-scanning parallel direction) resolution and resolution in $\mathrm{X}$ (scanning) direction $<2.2 \mu \mathrm{m}$ preserving an excellent phase stability in both directions [(Fig. 3(a), 3(b)].

The sensitivity (phase stability) and the associated error of the path length difference of the system determined by standard deviation over 200 successive frames on the mirror surface were measured with $510 \mathrm{pm}$ in accordance with previous reports [6].

\section{Measurements of red blood cells}

A sample of blood was obtained from a healthy donor and prepared on a microscope glass plate producing a blood smear. A typical human red blood cell (RBC) has a disk diameter of $6-8 \mu \mathrm{m}$, a thickness of $2 \mu \mathrm{m}$ and preserves a characteristic biconcave shape. The sample was exposed to an incident power of $1.4 \mathrm{~mW}$. Fig 3(a) shows an en-face reflectivity map of the blood smear taken at the maximum intensity of the front reflex from the glass surface underneath. Fig. 3(b) shows the reflectivity map of a selected RBC. Due to high transversal stability, we obtain the corresponding lateral phase image in Fig. 3(c) with no additional phase correction in post-processing except phase unwrapping and background subtraction [6]. Note that this phase map shows an accumulated phase change corresponding to the optical path length through the selected RBC. 

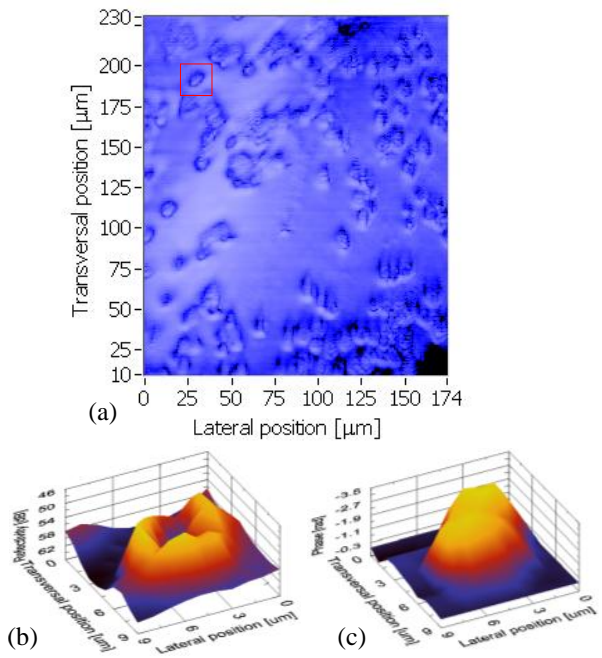

Fig. 3. (a) en-face reflectivity map of a blood smear, (b) 3D reflectivity image and (c) 3D phase map of a single RBC [indicated by red rectangle in (a)].

\section{Dispersion Contrast}

Sample dispersion is a physical parameter that contains valuable information of biochemical constitution and concentrations. Due to its characteristic change over the wavelength range it can be regarded as a spectroscopic molecularly sensitive contrast parameter. Complex interferometry allows accessing the sample dispersion as it is contained in the signal phase.

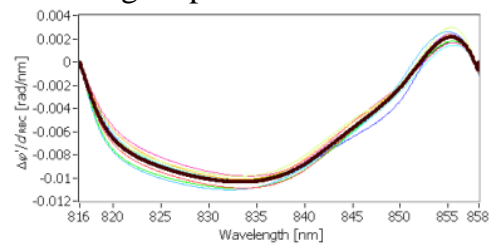

Fig. 4. Group delay and RBC height corrected phase curves (colored lines) within a region of a RBC [Fig. 3(c)]. The mean value of $\Delta \varphi^{\prime}(\lambda, x, y) / d_{\mathrm{RBC}}(x, y)$ is plotted as solid black line. The characteristic shape is clearly visible.

It has been demonstrated how spectral dispersion curves enable identification and quantification of different metabolite solutions such as glucose and albumin in a mixture [8]. The spectral phase is extracted at a single interface underneath the sample by using a Hilbert transform on the spectral OCT signal. However, the need of taking the second derivative for accessing the second order dispersion increases the noise level dramatically and the originally high sensitivity may be lost. Thus, if one is mainly interested in the characteristic shape of the spectral phase curve it should be sufficient to subtract the linear phase term, which is related to the group refractive index. The remaining higher order phase terms will then have a shape that could be used as a spectroscopic label-free molecularly specific contrast parameter. The group delay corrected phase $\left(\Delta \varphi^{\prime}(k, x, y)\right)$ is obtained by subtracting the linear fit of the phase $\left(\varphi_{\text {lin }}\right)$ at the central wavelength of the light source spectrum:

$\Delta \varphi^{\prime}(k, x, y)=\varphi-\varphi_{\text {lin }}-\varphi_{B G}=2 k n(k) \cdot d_{R B C}(x, y)$, where $k$ is the wave number, $n(k)$ is the refractive index, $d_{\mathrm{RBC}}(x, y)$ is the local thickness of the sample, and $\varphi_{\mathrm{BG}}$ is the reference offset phase. The group delay and RBC height corrected spectral phase curves $\left.\left(\Delta \varphi^{\prime}(\lambda, x, y)\right) / d_{\mathrm{RBC}}(x, y)\right)$ for a small region of the RBC in Fig.3 is shown in Fig. 4. We can clearly observe a specific spectroscopic signature over the wavelength range independent from the thickness of the sample with only a small deviation from the mean curve (solid black line). The spectral range for analyzing the phase from $816 \mathrm{~nm}$ to $858 \mathrm{~nm}$ was chosen so that the spectral intensity stayed above $1 / 10$ of the peak intensity. This way the spectral phase error is kept to $18-25 \mathrm{mrad}$ across the spectral range. At present, the spectroscopic dispersion signature is limited by the small bandwidth of used SLD to $\sim 40 \mathrm{~nm}$.

In this paper we demonstrate an important extension of our previously reported work - line field OCM. We demonstrate a lateral resolution of $\sim 2 \mu \mathrm{m}$ preserving excellent phase stability in both lateral directions. The above mentioned advantages of LF OCM were demonstrated by analyzing individual human red blood cells. The extraction of the accumulated spectral phase through the RBC allows producing a characteristic spectroscopic signature related to sample dispersion. The label-free dispersion contrast might impact on the diagnosis of various RBC diseases, but can similarly be used in micro-fluidics as a revolutionary technology for molecular biology like enzymatic analysis, DNA analysis, and proteomics. In addition, the high-speed capability of parallel FD-OCM offers potentially in vivo visualization of rapid dynamic processes on the cellular scale.

\section{References}

[1] W. Drexler, J.G. Fujimoto, Optical Coherence Tomography: Technology And Applications (Springer, 2008).

[2] J.A. Izatt, M.R. Hee, G.M. Owen, E.A. Swanson, J.G. Fujimoto, Opt. Lett. 19, 590 (1994).

[3] A. Dubois, K. Grieve, G.Moneron, R. Lecaque, L. Vabre, C. Boccara, Appl. Opt. 43, 2874 (2004).

[4] B. Grajciar, M. Pircher, A. F. Fercher, R. A. Leitgeb, Opt. Exp. 13, 1131 (2008), http://www.opticsinfobase.org/oe/abstract.cfm?URI=oe13-4-1131.

[5] Y. Chen, S.W. Huang, A.D. Aguirre, J.G. Fujimoto, Opt. Lett. 32, 1971 (2007).

[6] B. Grajciar, Y. Lehareinger, A.F. Fercher, R.A. Leitgeb, Opt. Exp. 18, 21841 (2010), http://www.opticsinfobase.org/oe/abstract.cfm?URI=oe18-21-21841.

[7] M. Choma, A. K. Ellerbee, Ch. Yang, T.L. Creazzo, J.A. Izatt, Opt. Lett. 30, 1162 (2005).

[8] S.M.Bagherzadeh, B. Grajciar, C.K. Hitzenberger, M. Pircher, A.F. Fercher, Opt. Lett. 32(20), 2924 (2007). 